\title{
Reducción del drenaje profundo y la lixiviación de nitrógeno en rotaciones agrícolas con cultivos de cobertura
}

\author{
Silvina I. Portela ${ }^{1, \infty}$; Silvina B. Restovich; Hugo M. González \& María J. Torti ${ }^{3}$ \\ ${ }^{1}$ Grupo Gestión Ambiental, EEA Pergamino, INTA. Pergamino, Buenos Aires, Argentina. ${ }^{2}$ Centro de Bioinvestigaciones, \\ Universidad Nacional del Noroeste de la Provincia de Buenos Aires y CONICET. Pergamino, Buenos Aires, Argentina. \\ ${ }^{3}$ LaboratorioRegionaldeCalidaddeAlimentos,Suelosy Aguas. EEAPergamino, INTA. Pergamino, Buenos Aires, Argentina.
}

Resumen. Los cambios de vegetación pueden alterar los flujos de agua y nitrógeno $(\mathrm{N})$ de los ecosistemas. La intensificación de las rotaciones con cultivos de cobertura (CC) otoño-invernales reduce el $\mathrm{N}$ del suelo y el riesgo de lixiviación durante los excesos hídricos. El objetivo de este trabajo fue contrastar el drenaje profundo y la lixiviación de $\mathrm{N}$ en una rotación soja-maíz con y sin CC, utilizando lisímetros. Se cultivó avena antes de soja y avena+vicia antes de maíz. Las precipitaciones fueron 732 y $562 \mathrm{~mm}$ durante los períodos intercultivo 2014 y 2015, y 817 y 705 mm durante los cultivos de soja y maíz, respectivamente. La inclusión de CC redujo el drenaje durante los períodos intercultivo (de 41 a $25 \%$ y de 22 a $9 \%$ de la precipitación durante el primer y segundo período intercultivo, respectivamente) y durante el cultivo de soja (de 18 a 14\% de la precipitación). Durante el cultivo de maíz, el drenaje fue similar en las dos rotaciones; representó el $14 \%$ de la precipitación. La concentración media de $\mathrm{N}-\mathrm{NO}_{3}{ }^{3}$ del drenaje fue de 6 y $15 \mathrm{mg} / \mathrm{L}$ para la rotación con y sin CC, respectivamente. La inclusión de CC redujo la lixiviación de 35 a 14 y de 17 a $3 \mathrm{~kg}$ N/ha durante los períodos intercultivo 2014 y 2015, respectivamente, y de 36 a 3 y de 15 a $6 \mathrm{~kg}$ N/ha durante los cultivos de soja y maíz, respectivamente. Estas reducciones, sumadas al aporte adicional de $\mathrm{N}$ por fijación biológica de vicia, produjeron balances de $\mathrm{N}$ positivos o cercanos a neutro, mientras que la rotación sin CC presentó balances negativos. Además de mejorar la circulación interna de $\mathrm{N}$, las reducciones en el drenaje con CC se pueden traducir en menor recarga del acuífero y contribuir a la regulación de inundaciones durante períodos lluviosos.

[Palabras clave: lisímetros de relleno, balance hídrico, balance de nitrógeno, nivel freático]

\begin{abstract}
Aвstract. Deep drainage and nitrogen leaching reduction in crop rotations with cover crops. Ecosystem water and nitrogen $(\mathrm{N})$ fluxes can be altered by vegetation changes. The intensification of crop rotations with fall-winter cover crops (CC) reduces soil $\mathrm{N}$ and leaching risk during period of water excess. In this study we used filled-in lysimeters to evaluate the effect of including CC (oats and an oats+vetch mixture) in a soybean-maize rotation on deep drainage and N leaching during a rainy period. Rainfall was 732 and 562 mm during 2014 and 2015 intercropping periods, and 817 and $705 \mathrm{~mm}$ during soybean and maize cropping periods, respectively. The introduction of CC reduced drainage during both intercropping periods (from 41 to $25 \%$ and from 22 to $9 \%$ of rainfall during the first and second intercropping periods, respectively) and during soybean cropping period (from 18 to $14 \%$ of rainfall). Drainage represented $14 \%$ of the rainfall of maize in both rotations. Mean drainage N-NO3- concentration was 6 and $15 \mathrm{mg} / \mathrm{L}$ for the rotation with and without CC, respectively, and the introduction of CC reduced leaching from 35 to 14 and from 17 to $3 \mathrm{~kg} \mathrm{~N} / \mathrm{ha}$ during 2014 and 2015 intercropping periods, respectively, and from 36 to 3 and from 15 to $6 \mathrm{~kg}$ N/ha during soybean and maize cropping periods, respectively. These reductions, together with the additional $\mathrm{N}$ input from biological fixation of vetch, produced positive or close to neutral $\mathrm{N}$ balances while the rotation without CC produced negative balances. The inclusion of CC in the rotation not only improved internal $\mathrm{N}$ circulation. The reduction of drainage can translate into less groundwater recharge and the concomitant contribution to flooding regulation during rainy periods.
\end{abstract}

[Keywords: filled-in lysimeters, water balance, nitrogen balance, water table level]

\section{INTRODUCCIÓN}

Los ciclos hidrológico y biogeoquímicos son más abiertos que cerrados a nivel de pequeñas cuencas, donde una fracción importante de lluvia y nutrientes se pierde por escurrimiento y drenaje profundo, y otra relativamente menor se acumula en el sistema (Perakis 2002). En general, los procesos bióticos asociados a las plantas y los microorganismos del suelo

Editor asociado: Jobbágy Esteban

$\triangle$ portela.silvina@inta.gob.ar cierran estos ciclos, especialmente los de los nutrientes esenciales como el nitrógeno $(\mathrm{N})$, a través de la absorción y el almacenamiento.

El $\mathrm{N}$ disponible es asimilado rápidamente por las plantas y transformado en compuestos orgánicos complejos que los microorganismos del suelo liberan, a través de la descomposición de la materia orgánica (MO), para ser reutilizado. De este modo, el N estaría

Recibido: 26 de abril de 2016

Aceptado: 23 de agosto de 2016 
fuertemente retenido y sujeto a muy pocas pérdidas durante la mayor parte del tiempo. Sin embargo, existen vías por las cuales se puede perder y que escapan al control que ejercen las plantas y los microorganismos (Perakis 2002; Perakis and Hedin 2002). Una vía de pérdida importante en agroecosistemas está asociada a las asincronías temporales y/o espaciales entre la oferta y la demanda del $\mathrm{N}$ utilizable por las plantas.

El balance de $\mathrm{N}$ (aplicado como fertilizante y/o fijado biológicamente - cosechado) a nivel de lote o región es valioso para estimar excedentes y pérdidas potenciales (Goulding 2004) ya que la cantidad de $\mathrm{N}$ que se lixivia es directamente proporcional a la magnitud del excedente (Hatch et al. 2003). Sin embargo, aun balances neutros o negativos pueden estar asociados a pérdidas cuando la oferta y la demanda de $\mathrm{N}$ no coinciden en el tiempo o en el espacio (Beaudoin et al. 2004). Por otro lado, el flujo de agua determina la pérdida potencial de N. Molenat et al. (2008) demostraron en tres cuencas agrícolas del oeste de Francia que la exportación de nitrato $\left(\mathrm{NO}_{3}^{-}\right)$hacia arroyos es un proceso limitado por la capacidad de transporte, que depende de cuánta agua fluye de manera superficial y subsuperficial hacia los cursos de agua.

Los cambios de vegetación pueden alterar la partición de la precipitación incidente en vapor (como evapotranspiración) y líquido (escurrimiento superficial y drenaje profundo). Nosetto et al. (2012) demostraron que el reemplazo de bosques secos o pastizales por monocultivo de soja reduce la evapotranspiración de 81 y $69 \%$ a $61 \%$ de la precipitación, respectivamente, y aumenta el escurrimiento superficial y el drenaje profundo (rendimiento hídrico). Un contraste menos extremo como la incorporación del cultivo de trigo antes de soja redujo la recarga primaveral del acuífero respecto del barbecho otoño-invernal seguido de un único cultivo anual estival (Portela 2012). Esto tiene consecuencias no sólo sobre la mejora en la eficiencia del sistema, sino también sobre la reducción de procesos que degradan el ambiente. Los excesos de agua de lluvia que no son aprovechados por la vegetación suelen ser responsables de problemas ambientales como la erosión hídrica y el escurrimiento y lavado de nutrientes (Sasal et al. 2010), de MO y de residuos de agroquímicos hacia cursos de agua superficiales y subterráneos (Caviglia et al. 2012).
En la Pampa Húmeda argentina, los sistemas agrícolas avanzaron en las últimas tres décadas hacia rotaciones simplificadas, con predominio de cultivos de verano (principalmente soja y, en segundo lugar, maíz) y períodos largos de barbecho otoño-invernal. Estos sistemas de cultivo son poco eficientes en la captura anual de precipitación y radiación solar (Caviglia et al. 2004) y presentan balances de $\mathrm{N}$ negativos, en particular en zonas de alto rendimiento y clima húmedo, asociados a las bajas dosis de fertilizantes nitrogenados (Álvarez et al. 2014; Austin et al. 2006). Por otro lado, el contenido elevado de MO de los suelos y las condiciones climáticas favorables de la región estimulan la producción de $\mathrm{NO}_{3}{ }_{3}$ a través de la mineralización de la $\mathrm{MO}$ nativa del suelo a lo largo del año. Esto representa una contribución significativa de $\mathrm{N}$ para los cultivos y una fuente de $\mathrm{N}$ lixiviable (Portela et al. 2006).

La intensificación de las rotaciones a partir de la inclusión de especies de ciclo otoñoinvernal como cultivos de cobertura (CC) reduce el $\mathrm{N}$ del suelo a través de su absorción e inmovilización, lo cual disminuye el riesgo de lixiviación durante los períodos de exceso hídrico (Constantin et al. 2010; Restovich et al. 2012; Thorup-Kristensen et al. 2003). La subsiguiente entrega del $\mathrm{N}$ absorbido por los CC (a partir de la descomposición de sus residuos), en sincronía con la demanda por parte del cultivo de cosecha siguiente, estimula el reciclaje de $\mathrm{N}$ en el sistema $\mathrm{y}$ permite mantener rendimientos similares o mayores a los obtenidos sin CC.

En este trabajo exploramos la intensificación de una rotación a partir del uso de CC como estrategia para aumentar la proporción de agua de lluvia evapotranspirada $y$, de este modo, reducir el rendimiento hídrico y la lixiviación, mejorando la circulación interna y retención de $\mathrm{N}$ en el sistema. Para ello realizamos mediciones directas de drenaje profundo y lixiviación de $\mathrm{N}$ en una rotación soja-maíz con y sin CC, a partir de lisímetros de relleno, y estimamos el balance de $\mathrm{N}$ de cada rotación.

\section{Materiales y MÉTOdOS}

Se utilizaron seis lisímetros de relleno (3.3x3.3x1.4 $\mathrm{m}$ de profundidad) construidos entre 1969 y 1970 en la EEA Pergamino del Instituto Nacional de Tecnología Agropecuaria (INTA) (3351' S, 6040’ O), y rellenados con dos suelos característicos de la Región Pampeana: 
un Argiudol típico de la serie Pergamino y un Hapludol típico de la serie Junín (tres de cada uno) (Soil Survey Staff 1999). Se reconstruyeron los perfiles de suelo hasta $1.2 \mathrm{~m}$ de profundidad colocando de forma secuencial cada horizonte y compactándolo hasta su densidad original sobre una capa de $0.12 \mathrm{~m}$ de arena y $0.04 \mathrm{~m}$ de grava que deriva el drenaje profundo hacia un tubo colector ubicado a $1.4 \mathrm{~m}$ de profundidad.

Los suelos pertenecen al orden de los Molisoles. Los Argiudoles predominan en la Pampa Ondulada. Son suelos profundos, bien desarrollados y bien drenados, con una secuencia de horizontes A-B-C bien definida y un horizonte Bt fuertemente argílico. Los Hapludoles son característicos de la zona de transición hacia la Pampa Interior. Son suelos bien drenados, con un horizonte $\mathrm{B}$ con menor proporción de arcilla y estructura más débil que el de los Argiudoles (Moscatelli 1991). Las características físicas y químicas de estos suelos se presentan en la Tabla 1.

El clima es templado húmedo sin estación seca, con temperatura media de 9.2 y $23.5{ }^{\circ} \mathrm{C}$ durante los meses más frío y más cálido, respectivamente (Hall et al. 1992). La precipitación media anual para el período 1910-2015 fue de $984 \mathrm{~mm}$, con un coeficiente de variación del $23 \%$ para las lluvias anuales del mismo período y una ocurrencia del 67\% del total de precipitaciones entre octubre y marzo (primavera y verano) (Red Agroclimática, INTA). Sin embargo, las precipitaciones durante este estudio, años 2014 y 2015, fueron 1695 y 1407 mm/año, respectivamente.

Los lisímetros estuvieron bajo agricultura continua durante 30 años, lo que le permitió a los suelos recuperar su estructura natural. En 2001 se inició una rotación maíz-soja bajo siembra directa utilizando las prácticas agronómicas típicas de la región. En 2014 se incorporaron CC entre los cultivos de verano: un lisímetro con cada suelo para la rotación con CC y dos para la rotación sin CC. Se cultivó avena (Avena sativa L.) como CC antecesor de soja y una consociación de avena y vicia (Vicia sativa L.) como antecesor de maíz (Tabla 2). Los CC se sembraron en línea bajo siembra directa utilizando densidades de $80 \mathrm{~kg}$ semilla/ha para avena y de $20+40 \mathrm{~kg}$ semilla/ha avena+vicia. La vicia fue inoculada con Rhizobium leguminosarum biovar. viceae inmediatamente antes de la siembra. Los CC no fueron ni cosechados, ni

Tabla 1. Características físicas y químicas de los suelos contenidos en los lisímetros: un Argiudol típico (serie Pergamino) y un Hapludol típico (serie Junín), representativos de los suelos de la Pampa Ondulada y de la zona de transición hacia la Pampa Interior, respectivamente. Las concentraciones de C, N y P corresponden al 2008.

Table 1. Physical and chemical characteristics of the soils contained in the lysimeters: a Typic Argiudoll (Pergamino series) and a Typic Hapludoll (Junín series), predominant in the Rolling Pampa and in the transition zone to the Inland Pampa, respectively. Carbon, N and P concentrations correspond to 2008.

\begin{tabular}{|c|c|c|c|c|c|c|c|c|c|}
\hline \multirow[b]{2}{*}{ Profundidad } & \multirow[b]{2}{*}{$\mathrm{m}$} & \multicolumn{4}{|c|}{ Argiudol típico } & \multicolumn{4}{|c|}{ Hapludol típico } \\
\hline & & $0-0.05$ & $0.05-0.30$ & $0.30-1.00$ & $1.00-1.20$ & $0-0.05$ & $0.05-0.30$ & $0.30-1.00$ & $1.00-1.20$ \\
\hline Horizonte & & \multicolumn{2}{|c|}{$\mathrm{A}+\mathrm{BA}$} & $\mathrm{Bt}$ & $\mathrm{BC}$ & \multicolumn{2}{|c|}{ A } & B & C \\
\hline Arcilla & $\%(w / w)$ & 21.6 & 20.6 & 38.8 & 19.0 & 10.5 & 19.2 & 18.0 & 10.4 \\
\hline Limo & & 58.4 & 55.6 & 43.5 & 55.9 & 39.5 & 31.8 & 33.4 & 30.6 \\
\hline Arena & & 20.0 & 23.8 & 17.7 & 25.1 & 50.0 & 49.0 & 48.6 & 59.0 \\
\hline Textura & & \multicolumn{2}{|c|}{ Franco limosa } & $\begin{array}{l}\text { Franco } \\
\text { arcillo } \\
\text { limosa }\end{array}$ & $\begin{array}{l}\text { Franco } \\
\text { limosa }\end{array}$ & \multicolumn{4}{|c|}{ Franco arenosa } \\
\hline Densidad aparente & $\mathrm{g} / \mathrm{cm}^{3}$ & 1.10 & 1.30 & 1.35 & 1.25 & 1.10 & 1.21 & 1.29 & 1.25 \\
\hline $\mathrm{CC}$ & $\%(w / w)$ & 28.9 & 27.0 & 27.6 & 25.4 & 23.8 & 23.5 & 19.8 & 15.7 \\
\hline PMP & & 14.6 & 15.4 & 18.5 & 13.9 & 10.9 & 8.9 & 9.5 & 9.0 \\
\hline $\mathrm{pH}_{\text {agua }}$ & $(1: 2.5)$ & 6.2 & 6.6 & 6.9 & 7.6 & 6.3 & 6.4 & 6.7 & 7.4 \\
\hline C orgánico & $\mathrm{g} / \mathrm{kg}$ & 26.3 & 15.8 & 5.5 & 4.3 & 23.1 & 14.6 & 5.6 & 2.4 \\
\hline $\mathrm{N}$ orgánico & & 2.34 & 1.51 & 0.94 & 0.88 & 2.09 & 1.37 & 0.89 & 0.53 \\
\hline P extractable & $\mathrm{mg} / \mathrm{kg}$ & 30.0 & 30.0 & 22.3 & 11.3 & 29.0 & 29.0 & 24.0 & 19.7 \\
\hline $\mathrm{T}$ & $\mathrm{cmol} / \mathrm{kg}$ & 21.0 & 20.4 & 30.8 & 25.6 & 14.4 & 15.0 & 13.1 & 12.0 \\
\hline
\end{tabular}

CC y PMP: contenido de agua en capacidad de campo y punto de marchitez permanente, respectivamente; T: capacidad de intercambio catiónico. 
Tabla 2. Secuencia de cultivos de los lisímetros.

Table 2. Cropping calendar, rainfall, fertilization and yield of crops grown in the lysimeters.

\begin{tabular}{cccccc}
\hline Cultivo & Siembra & $\begin{array}{c}\text { Cosecha o } \text { secado }^{3} \\
\text { Maíz }\end{array}$ & $\begin{array}{c}\text { Precipitación }^{4} \\
(\mathrm{~mm})\end{array}$ & $\begin{array}{c}\text { Fertilización }^{5} \\
(\mathrm{~kg} \mathrm{~N} / \mathrm{ha})\end{array}$ & $\begin{array}{c}\text { Rendimiento } \\
(\mathrm{Mg} / \mathrm{ha})(\% \text { humedad })\end{array}$ \\
\hline Avena $^{1}$ & $7 / 10 / 2013$ & $18 / 3 / 2014$ & 898 & $57-84$ & $12.2(14.5 \%)$ \\
Soja & $7 / 18 / 5 / 2014^{2}$ & $17 / 10 / 2014$ & $320(732)$ & - & 8,6 \\
Avena+vicia $^{1}$ & $24 / 4 / 2015$ & $24 / 4 / 2015$ & 817 & - & $5.0(13.5 \%)$ \\
Maíz & $5 / 10 / 2015$ & $24 / 8 / 2015$ & $477(562)$ & - & 7,2 \\
& & $23 / 3 / 2016$ & 705 & $57-84$ & $10.2(14.5 \%)$ \\
\hline
\end{tabular}

\footnotetext{
${ }^{1}$ Sólo en los lisímetros con la rotación con cultivos de cobertura

${ }^{2}$ Siembra y resiembra, respectivamente

${ }^{3}$ Para los cultivos de cosecha y de cobertura, respectivamente

${ }^{4}$ El valor entre paréntesis representa la lluvia del período de barbecho completo

${ }^{5}$ Para el Argiudol típico y Hapludol típico, respectivamente
}

pastoreados, ni incorporados al suelo, y su ciclo se interrumpió de forma química con 3-4 L/ha de glifosato ( $48 \%$ principio activo). La fecha de secado se definió en función de la fecha de siembra del cultivo siguiente y de la distribución de las lluvias: antes de soja, se secaron en primavera (octubre), durante la etapa reproductiva, y antes de maíz se secaron en invierno (agosto), en estado vegetativo.

El maíz (Zea mays L.) DK 747 (Monsanto) se sembró en líneas separadas a $0.60 \mathrm{~m}$ y con $0.20 \mathrm{~m}$ entre plantas (80000 plantas/ha). En el momento de la siembra se realizó una fertilización fosforada de base con superfosfato triple (24 $\mathrm{kg} \mathrm{P}_{2} \mathrm{O}_{5} / \mathrm{ha}$ ) y en cuarta hoja se fertilizó por voleo con urea. Las dosis de fertilizante (57 y $84 \mathrm{~kg} \mathrm{~N} / \mathrm{ha}$, para Pergamino y Junín, respectivamente) se calcularon a partir del método del balance, asumiendo una demanda de $261 \mathrm{~kg} \mathrm{~N} /$ ha para un rendimiento objetivo de $11 \mathrm{Mg} / \mathrm{ha}$, una oferta de $\mathrm{N}$ del suelo de 204 y $177 \mathrm{~kg} / \mathrm{ha}$ para Pergamino y Junín, respectivamente (contenido inicial de $\mathrm{N}-\mathrm{NO}_{3}{ }^{-}+\mathrm{N}^{-\mathrm{NO}_{3}}$ - producido por mineralización del $\mathrm{N}$ orgánico del suelo durante el ciclo del cultivo) y una eficiencia de uso del fertilizante de $75 \%$. Las malezas se controlaron en preemergencia con atrazina y acetochlor $(2 \mathrm{~kg} /$ ha de cada uno) y en post-emergencia con halosulfuron (130 g/ha) y foramsulfuron (120 $\mathrm{g} / \mathrm{ha}$ ). El cultivo siguiente de la rotación fue soja (Glycine max (L) Merr.) var. Don Mario 5009, sembrada en líneas espaciadas a 0.52 m (500000 plantas/ha). La soja se inoculó con Bradyrhizobium sp. y no se fertilizó.

La biomasa aérea de los CC fue medida en el momento del secado, cosechando 0.25 $\mathrm{m}^{2}$ por lisímetro. El material cosechado fue secado en estufa a $65{ }^{\circ} \mathrm{C}$. Se determinó el peso seco y se extrajo una submuestra para medir el contenido total de $\mathrm{N}$ por Kjeldahl. El rendimiento de maíz y de soja se obtuvo a partir de la cosecha de la superficie total de cada lisímetro $\left(10 \mathrm{~m}^{2}\right)$. A excepción del grano, todo el material vegetal fue redistribuido sobre la superficie de los lisímetros para simular el sistema de labranza cero.

La precipitación se registró diariamente en la estación meteorológica de la EEA, ubicada a $30 \mathrm{~m}$ de los lisímetros. Después de cada evento de lluvia que produjo drenaje se midió diariamente su volumen hasta que cesó el drenaje. Para ello se emplearon recipientes ubicados a la salida de los tubos colectores de cada lisímetro. De cada drenaje se extrajo una muestra de $200 \mathrm{~mL}$ para determinar la concentración de $\mathrm{NO}_{3}^{-}$por el método del fenol disulfónico (Mulvaney 1996). Las pérdidas por lixiviación se calcularon para cada recolección como el producto del volumen drenado por su concentración de $\mathrm{NO}_{3}^{-}$y se expresaron en $\mathrm{kg} \mathrm{N} / \mathrm{ha}$. La concentración media de $\mathrm{NO}_{3}$ se calculó para cada período de cultivo o intercultivo y para el ciclo completo de rotación soja-maíz como el cociente entre la sumatoria del $\mathrm{N}$ lixiviado y la sumatoria del drenaje, y se expresó en mg N/L.

Se calculó un balance de $\mathrm{N}$ para cada año y rotación. Se consideraron como ingresos a la fertilización nitrogenada, la fijación biológica de las leguminosas y la deposición atmosférica, y como egresos al $\mathrm{N}$ exportado con la cosecha de grano y al $\mathrm{N}$ lixiviado. Se utilizó la relación entre el $\mathrm{N}$ de la biomasa y el $\mathrm{N}$ fijado biológicamente para estimar el N fijado por soja (Salvagiotti et al. 2008). El $\mathrm{N}$ de la biomasa aérea se estimó a partir del rendimiento en grano, y el $\mathrm{N}$ de la biomasa total (aérea y subterránea) se calculó asumiendo que $24 \%$ del $\mathrm{N}$ total se encuentra 
en las raíces. En el caso de vicia, se estimó el $\mathrm{N}$ fijado biológicamente a partir de la relación entre la biomasa y el $\mathrm{N}$ fijado desarrollada para alfalfa (Álvarez et al. 2014). El ingreso de $\mathrm{N}$ por deposición atmosférica se estimó a partir de las concentraciones de amonio y nitrato de muestras de lluvia recolectadas mensualmente en Pergamino en el marco de la Red de Deposiciones Atmosféricas del Río de la Plata (Carnelos et al. 2014) y del volumen de lluvia registrado en la estación meteorológica. El N exportado en el grano se estimó a partir del rendimiento y del porcentaje de $\mathrm{N}$ del grano: $1.5 \%$ para maíz (Echeverría and Sainz Rozas 2006) y 6.34\% para soja (Salvagiotti et al. 2008).

\section{Análisis estadístico}

El diseño experimental fue en bloques completamente aleatorizados, con dos repeticiones para la rotación con CC y cuatro repeticiones para la rotación sin CC. Se consideró como bloque a cada tipo de suelo (Argiudol típico y Hapludol típico) y como tratamiento a cada rotación (avena-sojaavena+vicia-maíz y barbecho-soja-barbechomaíz). Para comparar el volumen drenado y el N lixiviado entre los tratamientos se utilizó el análisis de varianza con el procedimiento GLM de SAS (SAS 2008). La comparación de medias se realizó a través del test de Duncan $(P<0.01)$.

\section{Resultados}

Las precipitaciones fueron 732 y 562 $\mathrm{mm}$ durante el primer y segundo período intercultivo, de los cuales 320 y $477 \mathrm{~mm}$, respectivamente, ocurrieron durante el crecimiento de los CC. El primer año el drenaje representó el $41 \%$ de la precipitación y el segundo año representó el $22 \%$ en el caso de la rotación sin CC. Los CC redujeron estas

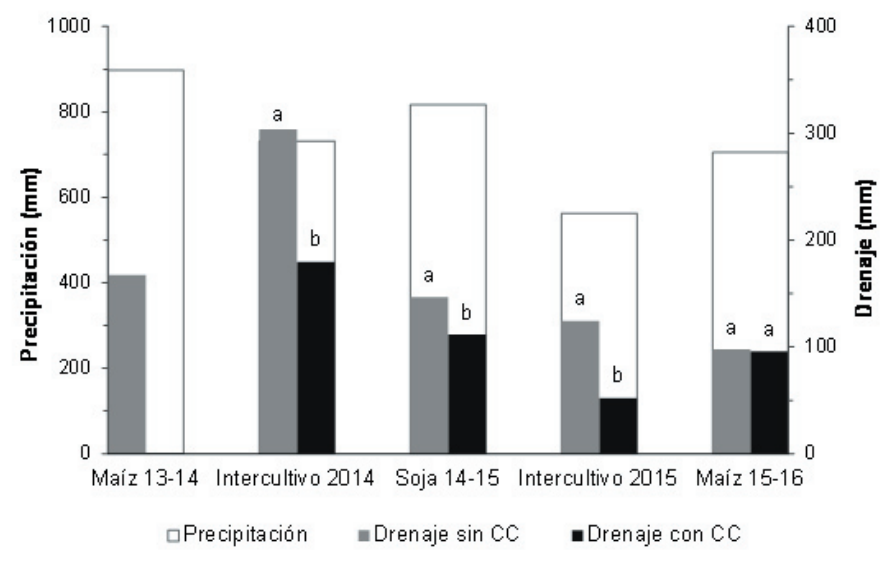

proporciones al 25 y 9\% de la precipitación de los mismos periodos. Durante el ciclo de soja, la precipitación fue de $817 \mathrm{~mm}$ y el drenaje representó 18 y 14\% de la misma en la rotación sin y con CC, respectivamente. Durante el ciclo de maíz la precipitación fue de $705 \mathrm{~mm}$ y el drenaje fue similar en las dos rotaciones, representando el $14 \%$ de la precipitación (Figura 1).

La concentración media de $\mathrm{N}-\mathrm{NO}_{3}{ }^{-}$del drenaje durante un ciclo completo de rotación soja-maíz fue de 6 y $15 \mathrm{mg} / \mathrm{L}$ para el tratamiento con y $\sin \mathrm{CC}$, respectivamente. La reducción en la lixiviación de $\mathrm{N}$ con $\mathrm{CC}$ varió entre 60 y 90\%: de 35 a 14 y de 17 a 3 kg $\mathrm{N} /$ ha durante los períodos intercultivo 2014 y 2015, respectivamente, y de 36 a 3 y de 15 a $6 \mathrm{~kg} \mathrm{~N} /$ ha durante los cultivos de soja y maíz, respectivamente (Figura 2).

Durante el primer ciclo de la rotación (barbecho o CC+soja) los ingresos de N (deposición atmosférica+fijación biológica de soja) fueron similares para los tratamientos con y sin CC (340 kg N/ha). El rendimiento en grano de soja fue similar entre la rotación con y sin CC y promedió $5.0 \mathrm{Mg} / \mathrm{ha}$, de modo que los egresos de $\mathrm{N}$ (exportación en grano+lixiviación) difirieron de la mano de la diferencia en lixiviación (341 y 402 kg N/ha para la rotación con y sin CC, respectivamente) (Tabla 3). Durante el segundo ciclo de la rotación (barbecho o CC+maíz), los ingresos de N (deposición atmosférica+fertilizante +fijación biológica de vicia) se duplicaron en el caso de la rotación con CC respecto de la rotación sin CC (167 vs. 76 kg N/ha) como consecuencia del ingreso adicional de $\mathrm{N}$ por fijación biológica en el caso de la consociación avena+vicia que se usó como CC. El rendimiento en grano de maíz fue similar entre la rotación con y sin CC (10.2 Mg/ha) al igual que los egresos de $\mathrm{N}$ correspondientes

Figura 1. Precipitación y drenaje profundo durante los períodos de cultivo e intercultivo para la rotación con y sin CC. El maíz anterior a la incorporación de CC a la rotación (maíz 13-14) se incluyó como referencia. Letras diferentes indican diferencias $(\mathrm{P}<0.01)$ entre la rotación con $C C$ y la rotación sin $\mathrm{CC}$ para cada período de cultivo o barbecho.

Figure 1. Rainfall and deep drainage during cropping and intercropping periods for the rotation with and without cover crop. The maize cultivated before the introduction of cover crops in the rotation (maize 13-14) was included as a reference. Different letters within each cropping or intercropping period indicate significant differences $(\mathrm{P}<0.01)$ between rotations. 


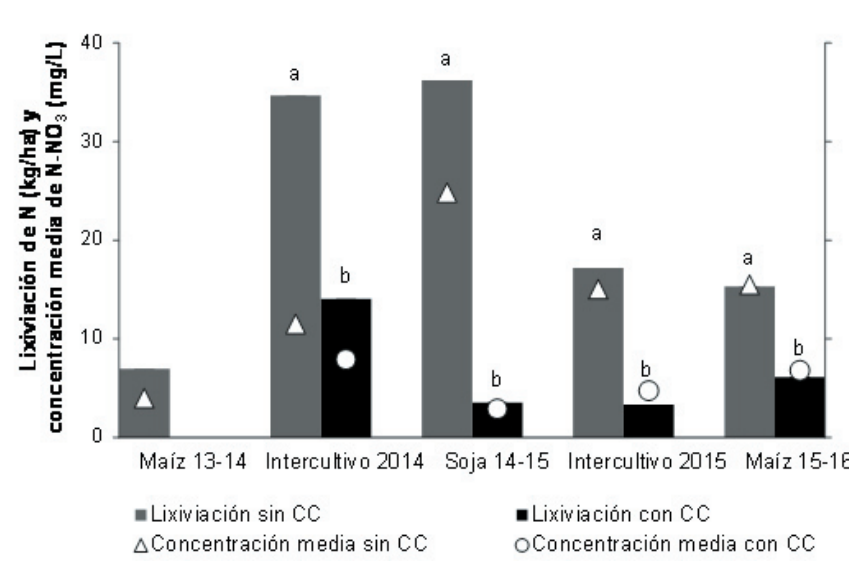

Figura 2. Lixiviación (barras) y concentración media de $\mathrm{N}^{-\mathrm{NO}_{3}}$ en el drenaje (símbolos) durante los períodos de cultivo e intercultivo para la rotación con y sin CC. El maíz anterior a la incorporación de CC a la rotación (maíz 1314) se incluyó como referencia. Letras diferentes indican diferencias de lixiviación $(P<0.01)$ entre la rotación con CC y la rotación sin CC para cada período de cultivo o barbecho.

Figure 2. Nitrogen leaching (bars) and drainage mean $\mathrm{N}-\mathrm{NO}_{3}^{-}$concentration (symbols) during cropping and intercropping periods for the rotation with and without cover crop. The maize cultivated before the introduction of cover crops in the rotation (maize 13-14) was included as a reference. Different letters within each cropping or intercropping period indicate significant differences $(P<0.01)$ between rotations.

Tabla 3. Componentes del balance de $\mathrm{N}(\mathrm{kg} / \mathrm{ha}$ ) para cada rotación y año.

Table 3. Nitrogen balance components $(\mathrm{kg} / \mathrm{ha})$ for each rotation and year.

\begin{tabular}{lcccccc}
\hline \multicolumn{1}{c}{ Rotación } & \multicolumn{3}{c}{ Ingresos } & \multicolumn{2}{c}{ Egresos } & Balance \\
\hline & $\begin{array}{c}\text { Deposición } \\
\text { atmosférica }\end{array}$ & Fertilizante & $\begin{array}{c}\text { Fijación } \\
\text { biológica }\end{array}$ & $\begin{array}{c}\text { Exportación } \\
\text { grano }\end{array}$ & Lixiviación & \\
Avena-soja & 6 & 0 & 329 & 323 & 18 & -5 \\
Barbecho-soja & 6 & 0 & 338 & 331 & 71 & -58 \\
Avena+vicia-maíz & 6 & 70 & 91 & 131 & 9 & 27 \\
Barbecho-maíz & 6 & 70 & 0 & 131 & 33 & -87 \\
\hline
\end{tabular}

${ }^{1}$ Corresponde al promedio de la dosis aplicada a los dos tipos de suelo

a la exportación en grano (131 kg N/ha), pero los egresos por lixiviación se triplicaron en la rotación sin CC (9 vs. $33 \mathrm{~kg} \mathrm{~N} / \mathrm{ha}$ ). En consecuencia, los cultivos de cobertura mejoraron el balance de $\mathrm{N}$ los dos años: durante el primero, la rotación con CC (avenasoja) produjo balance de $\mathrm{N}$ cercano a neutro mientras que la rotación sin CC (barbechosoja) tuvo balance negativo (-5 y $-58 \mathrm{~kg} \mathrm{~N} / \mathrm{ha}$, respectivamente) y durante el segundo año, la rotación con CC (avena+vicia-maíz) produjo balance de $\mathrm{N}$ positivo y la rotación sin $\mathrm{CC}$ (barbecho-maíz) tuvo balance negativo (27 y $-87 \mathrm{~kg} \mathrm{~N} /$ ha, respectivamente).

\section{DisCUSIÓN}

La intensificación de las rotaciones agrícolas con cultivos de cobertura otoño-invernales reduce el $\mathrm{N}$ del suelo respecto de las rotaciones que mantienen barbecho entre dos cultivos estivales (Restovich et al. 2012). Una vez completado su ciclo de crecimiento, los CC reducen entre 50 y $90 \%$ el $\mathrm{N}_{-} \mathrm{NO}_{3}^{-}$del suelo respecto del que permaneció en barbecho. A partir de mediciones directas de drenaje y lixiviación, en este trabajo demostramos que la captura de agua y $\mathrm{N}$ por los CC se traduce en reducciones equivalentes (60-90\%) en la lixiviación de $\mathrm{N}$ durante períodos con excesos hídricos.
La estación otoño-invernal (mayoseptiembre) que se propone intervenir con CC en reemplazo del barbecho, presenta precipitaciones que varían entre 100 y 300 mm para la Región Pampeana (la precipitación acumuladaen estosmesesen Pergaminosuperó los $350 \mathrm{~mm}$ sólo $9 \%$ de los años entre 1910 y 2015) y baja pluviometría por evento $(<10 \mathrm{~mm})$ (Caviglia et al. 2012). Sin embargo, las lluvias de los períodos intercultivo estudiados fueron muy superiores (732 y $562 \mathrm{~mm}$ representando 43 y $40 \%$ de los totales anuales), con un mes de mayo (2014) con el doble de lluvia que el promedio histórico (121 vs. $59 \mathrm{~mm}$ ) y un mes de agosto (2015) que presentó cinco veces la precipitación promedio (262 vs. $42 \mathrm{~mm}$ ). Re y Barros (2009) demostraron que entre 1959 y 2002 se produjo un cambio positivo en la intensidad de los eventos de lluvia extremos en la Región Pampeana, indicando que los picos de precipitación que captamos en los años analizados responden a una tendencia regional. Las elevadas precipitaciones de los dos años que abarcó este trabajo fueron responsables de importantes pérdidas de $\mathrm{N}$ a través del drenaje. Durante un ciclo completo de rotación soja-maíz se perdieron $103 \mathrm{~kg} \mathrm{~N} /$ ha a través de un drenaje acumulado de 672 $\mathrm{mm}$. En estos años, la intensificación de la rotación con CC de ciclo otoño-invernal redujo la pérdida de $\mathrm{N}$ a $27 \mathrm{~kg} / \mathrm{ha}$ con un drenaje 
acumulado de $439 \mathrm{~mm}$, demostrando que prolongar el período con cobertura vegetal es fundamental para cerrar los ciclos hidrológico y de $\mathrm{N}$, en particular durante períodos de excesos hídricos.

La cobertura vegetal regula el contenido de humedad del suelo a través de la transpiración y de la influencia del rastrojo en superficie sobre la evaporación, y la disponibilidad de agua de lluvia para recargar el acuífero (Mastrocicco et al. 2010; Zhang and Schilling 2006). La incorporación del cultivo de trigo antes de soja acorta el período de barbecho entre dos cultivos estivales y permite que una mayor proporción del agua del suelo sea transpirada, reduciendo la recarga (flujo de agua que llega a la superficie freática del acuífero) y el ascenso freático durante la primavera respecto del barbecho seguido de un único cultivo anual estival (Portela 2012). En este trabajo medimos reducciones en el drenaje a partir de la inclusión de CC en una rotación que se pueden traducir en 1.24 y $0.72 \mathrm{~m}$ menos de ascenso freático durante los barbechos 2014 y 2015, respectivamente, respecto de la rotación sin CC. En vastos sectores de la Llanura Pampeana, como resultado del clima húmedo a subhúmedo y el relieve regional plano con pobres redes de escurrimiento superficial, los niveles freáticos se encuentran cercanos a la superficie $(<5 \mathrm{~m}$ de profundidad) en gran parte del paisaje (Aradas et al. 2002). En sistemas naturales o cultivados con estas características, el aumento en la recarga asociado a períodos lluviosos puede resultar en ascensos del nivel freático que producen inundaciones en zonas bajas del paisaje (Forte Lay et al. 2007; Nosetto et al. 2009; Viglizzo et al. 2009). Si tenemos en cuenta que durante el período de este estudio el nivel freático osciló entre 0 y $1 \mathrm{~m}$ de profundidad en las posiciones de media loma y pie de loma (Red de Monitoreo de las Aguas y del Territorio 2015), la reducción de la recarga a partir del uso de CC contribuiría a la regulación de inundaciones. En la Región Pampeana, donde generalmente las obras hidráulicas poseen un impacto muy localizado (Menéndez et al. 2012), la elección y manejo de cultivos debería tenerse en cuenta como herramienta para influenciar la dinámica freática (Florio et al. 2015).

A pesar del aumento en el uso de fertilizantes nitrogenados (x8 entre 1990 y 2013), en la Región Pampeana húmeda no se llega a compensar la exportación de $\mathrm{N}$ de los principales cultivos (FAOSTAT 2013). En la campaña 2006/2007 se repuso sólo 50\% del N extraído por los cultivos de soja, trigo, maíz y girasol, y los suelos se están empobreciendo en materia y N orgánicos (García et al. 2009). En el caso de la soja, que ocupa más de $60 \%$ de la superficie total cultivada en Argentina, Austin et al. (2006) estimaron que el N proveniente de la fijación biológica tampoco es suficiente para compensar la exportación de N con el grano. Salvagiotti et al. (2008) introdujeron una modificación importante a la estimación del $\mathrm{N}$ fijado biológicamente por el cultivo de soja al contemplar el $\mathrm{N}$ de las raíces. Este cambio produjo balances de $\mathrm{N}$ apenas negativos o neutros. En este contexto podríamos asumir que el sistema de cultivo regional (con cultivos de verano y barbecho otoño invernal) no presenta excedentes de $\mathrm{N}$ y desestimar las pérdidas potenciales por lixiviación. Sin embargo, Portela et al. (2009) estimaron a partir de un modelo de simulación que el sistema de cultivo típico de la región produce un aporte de $\mathrm{N}$ del suelo al agua subterránea que varía entre 0 y 36 kg N-NO ${ }_{3}^{-} \cdot$ ha $^{-1}$.año ${ }^{-1}$, que es relativamente homogéneo a lo largo del paisaje.

Las pérdidas por lixiviación son rara vez tenidas en cuenta para los balances de $\mathrm{N}$ porque son difíciles de estimar. Los lisímetros de relleno son microecosistemas artificiales bien delimitados que permiten computar las entradas y salidas en forma prácticamente completa y sirven para generar información relevante acerca de los procesos de ciclado de nutrientes que, generalmente, no se puede obtener a partir aproximaciones ecosistémicas de gran escala (Lajtha 2000). Presentan, sin embargo, limitaciones estructurales como el tiempo necesario para la estructuración del suelo y un número limitado de repeticiones. En este trabajo realizamos mediciones directas de drenaje y lixiviación a partir de lisímetros. Estas mediciones nos permitieron concluir que las pérdidas por lixiviación están más vinculadas a la cantidad de agua que pasa por el suelo que al excedente de $\mathrm{N}$ calculado como la diferencia entre las entradas (fertilización nitrogenada, fijación biológica de las leguminosas y deposición atmosférica) y lo exportado con la cosecha de grano (Figura 3). Así, balances negativos pueden estar asociados a pérdidas por lixiviación cuando la oferta y la demanda de $\mathrm{N}$ no coinciden temporalmente y cuando el $\mathrm{N}$ lixiviado proviene de una fuente no controlada como la mineralización de la MO acumulada en el suelo y, en menor medida, del fertilizante nitrogenado residual (Portela et al. 2006). 


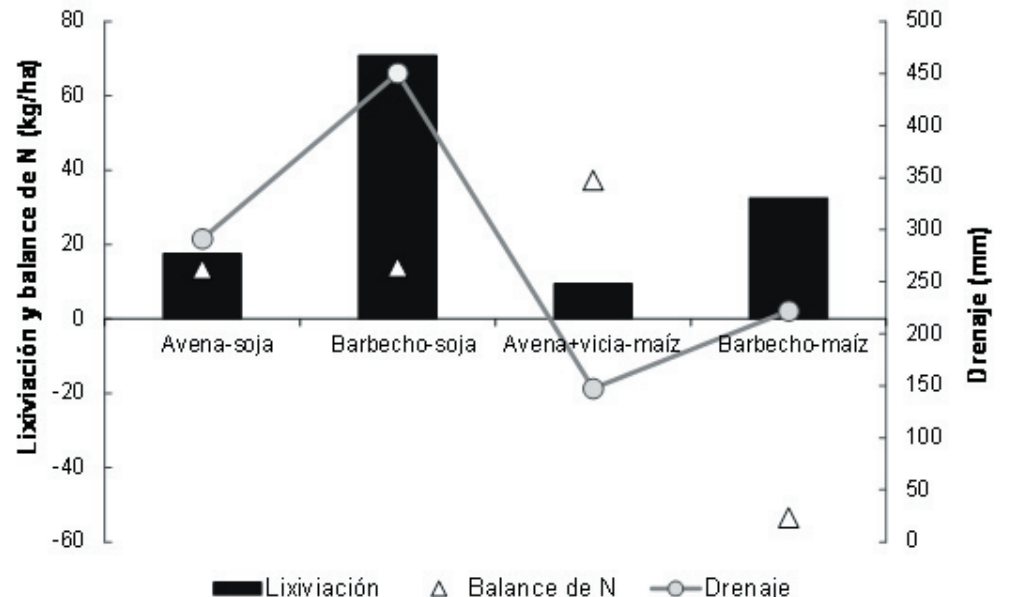

Figura 3. Lixiviación, balance de $\mathrm{N}$ y drenaje para la rotación con y sin CC. Para el balance se consideró como egreso sólo el $\mathrm{N}$ exportado con la cosecha de grano.

Figure 3. Nitrogen leaching, N balance and deep drainage for the rotation with and without cover crop. For the $\mathrm{N}$ balance, grain $\mathrm{N}$ exports were the only output considered.
En síntesis, en este trabajo demostramos que la inclusión de CC como antecesores de los cultivos de verano redujo significativamente las pérdidas de $\mathrm{N}$ por lixiviación: por un lado, a partir de la reducción del drenaje, y por otro, por la inmovilización de $\mathrm{N}$ en la biomasa vegetal. Al incluir la pérdida de $\mathrm{N}$ por lixiviación en el balance, estas reducciones, sumadas al aporte adicional de $\mathrm{N}$ por fijación biológica en el caso de la consociación de avena y vicia como CC, produjeron balances de $\mathrm{N}$ positivos o cercanos a neutro mientras que las rotaciones sin CC presentaron balances muy negativos. A partir de la mejora de los balances de $\mathrm{N}$ y de las reducciones en la concentración media de $\mathrm{N}-\mathrm{NO}_{3}{ }^{-}$en el drenaje, se desprende que la inclusión de CC aumenta la circulación interna de N. Este aumento en la retención de $\mathrm{N}$ debería redundar en mejoras en el stock de $\mathrm{N}$ orgánico del suelo en el mediano o largo plazo.

Agradecimientos. Este trabajo se realizó con el apoyo del Instituto Nacional de Tecnología Agropecuaria (INTA) a través del Proyecto PNAGUA 1133033 “Gestión del agua en producciones vegetales de secano". Agradecemos la revisión de P.I. Araujo que contribuyó a mejorar el contenido y la claridad del manuscrito y la ayuda de M.J. Beribe con el diseño experimental. También agradecemos a D. Colombini y F. Villalba por su ayuda con la recolección de datos y tareas de campo.

\section{REFERENCIAS}

Álvarez, R., H. S. Steinbach, and J. L. De Paepe. 2014. A regional audit of nitrogen fluxes in pampean agroecosystems. Agriculture, Ecosystems \& Environment 184:1-8.

Aradas, R., J. Lloyd, and J. Palmer. 2002. Groundwater problems in low elevation regional plains: the Buenos Aires province example. Pp. 613-623 en: Bocanegra, E., D. Martínez and H. Massone (eds.). Groundwater and human development. Mar del Plata, Buenos Aires.

Austin, A. T., G. Piñeiro, and M. González-Polo. 2006. More is less: agricultural impacts on the N cycle in Argentina. Biogeochemistry 79:45-60.

Beaudoin, N., B. Mary, F. Laurent, G. Aubrion, and J. K. Saad. 2004. Is the N balance a good indicator of nitrogen losses in arable systems? Pp. 487-489 en: D. J.Hatch, D. R. Chadwick, S. C. Jarvis and J. A. Roker (eds.). Controlling nitrogen flows and losses. Wageningen Academic Publishers. The Netherlands.

Carnelos, D. A., C. L. Michel, S. Portela, E. G. Jobbágy, R. B. Jackson, et al. 2014. Variación espacial y temporal de las deposiciones atmosféricas en Argentina y Uruguay. Reunión Binacional Uruguay-Argentina de Agrometeorología y XV Reunión Argentina de Agrometeorología. 1-3 octubre, Piriápolis, Uruguay.

Caviglia, O. P., V. O. Sadras, and F. H. Andrade. 2004. Intensification of agriculture in the south-eastern Pampas: I. Capture and efficiency in the use of water and radiation in double-cropped wheat-soybean. Field Crops Research 87(2-3):117-129.

Caviglia, O. P., S. Albarenque, C. Gregorutti, N. Van Opstal, and R. Melchiori. 2012. Cuál es el impacto de las alternativas invernales de intensificación sobre el balance hídrico del sistema? Revista técnica de la asociacion argentina de productores en siembra directa. Aapresid. Pp. 2-10.

Constantin, J., B. Mary, F. Laurent, G. Aubrion, A. Fontaine, et al. 2010. Effects of catch crops, no till and reduced nitrogen fertilization on nitrogen leaching and balance in three long-term experiments. Agriculture, Ecosystems and Environment 135(4):268-278.

Echeverría, H. E., and H. Sainz Rozas. 2006. Maíz. Pp. 255-282 en: H. E. Echeverría and F. O. García (eds.). Fertilidad de suelos y fertilización de cultivos. Editorial INTA. Buenos Aires, Argentina.

FAOSTAT. 2013. http:/ / faostat.fao.org/ 
Florio, E. L., J. L. Mercau, and M. D. Nosetto. 2015. Factores que regulan la dinámica freática en dos ambientes de la Pampa Interior con distintos regímenes de humedad. Ciencia del Suelo 33(2):263-272.

Forte Lay, J. A., E. Kruse, and J. L. Aiello. 2007. Hydrologic scenarios applied to the agricultural management of the northwest of the Buenos Aires Province, Argentina. GeoJournal 70(4):263-271.

García, F. O., I. A. Ciampitti, G. Rubio, and L. I. Picone. 2009. La fertilización fosfatada en la Argentina: Actualidad, manejo y perspectivas. Jornadas Nacionales “Sistemas Productivos Sustentables: Fósforo, Nitrógeno y Cultivos de Cobertura". Bahía Blanca, Argentina.

Goulding, K. 2004. Pathways and losses of fertilizer nitrogen at different scales. Pp. 209-219 en: Mosier, A. R., J. K. Syers and J. R. Freney (eds.). Agriculture and the nitrogen cycle. Island Press. Washington, USA.

Hall, A. J., C. M. Rebella, C. M. Ghersa, and J. P. Culot. 1992. Field-crop systems of the pampas. Pp. 413-450 en: Pearson, CJ (ed.). Field crop ecosystems. Ecosystems of the world. Elsevier. Amsterdam.

Hatch, D. K., W. T. Goulding, and D. V. Murphy. 2003. Nitrogen. Pp. 7-27 en: Haygarth, P. M. and S. C. Jarvis (eds.). Agriculture, hydrology and water quality. CABI Publishing. Wallingford.

Lajtha, K. 2000. Ecosystem nutrient balance and dynamics. Pp. 249-264 en: Sala, O. E., R. B. Jackson, H. A. Mooney and R. W. Howarth (eds.). Methods in ecosystem science. Springer-Verlag. New York.

Mastrocicco, M., N. Colombani, E. Salemi, and G. Castaldelli. 2010. Numerical assessment of effective evapotranspiration from maize plots to estimate groundwater recharge in lowlands. Agricultural Water Management 97(9):1389-1398.

Menéndez, N., N. Badano, E. Lecertua, F. Re, and M. Re. 2012. Evaluación de las inundaciones y las obras de drenaje en la cuenca del Salado (Prov. Buenos Aires) mediante modelación numérica. Proyecto LHA 331. Informe LHA 01331-12. Instituto Nacional del Agua. Subsecretaria de Recursos Hídricos. Secretaría de Obras Públicas. República Argentina.

Molenat, J., C. Gascuel-Odoux, L. Ruiz, and G. Gruau. 2008. Role of water table dynamics on stream nitrate export and concentration in agricultural headwater catchment (France). Journal of Hydrology 348(3-4):363-378.

Moscatelli, G. N. 1991. Los suelos de la región pampeana. Pp. en: Barsky, O. (ed.). El desarrollo agropecuario pampeano. INDEC-INTA-IICA. Buenos Aires.

Mulvaney, R. L. 1996. Nitrogen inorganic forms. Pp. 1123-1184 en: Sparks, D. L., A. L. Page, P. A. Helmke, R. H. Loeppert, P. N. Soltanpour et al. (eds.). Methods of soil analysis: Chemical methods. Part 3. Soil Science Society of America and American Society of Agronomy. Madison, Wisconsin, USA.

Nosetto, M. D., E. G. Jobbágy, R. B. Jackson, and G. A. Sznaider. 2009. Reciprocal influence of crops and shallow ground water in sandy landscapes of the Inland Pampas. Field Crops Research 113:138-148.

Nosetto, M. D., E. G. Jobbágy, A. B. Brizuela, and R. B. Jackson. 2012. The hydrologic consequences of land cover change in central Argentina. Agriculture, Ecosystems \& Environment 154(0):2-11.

Perakis, S. S. 2002. Nutrient limitation, hydrology and watershed nitrogen loss. Hydrological Processes 16:35073511.

Perakis, S. S., and L. O. Hedin. 2002. Nitrogen loss from unpolluted South American forests mainly via dissolved organic compounds. Nature 415:416-419.

Portela, S., A. Andriulo, M. Sasal, B. Mary, and E. Jobbágy. 2006. Fertilizer vs. organic matter contributions to nitrogen leaching in cropping systems of the Pampas: 15N application in field lysimeters. Plant and Soil 289(1-2):265-277.

Portela, S. I. 2012. Transferencia de nitrógeno de ecosistemas agrícolas recientemente intensificados a acuíferos: efecto de la variación en las precipitaciones. Tesis para optar al título de Doctor de la Universidad de Buenos Aires, Área Ciencias Agropecuarias. Facultad de Agronomía, Universidad de Buenos Aires. Pp. 110.

Portela, S. I., A. E. Andriulo, E. G. Jobbágy, and M. C. Sasal. 2009. Water and nitrate exchange between cultivated ecosystems and groundwater in the Rolling Pampas. Agriculture, Ecosystems \& Environment 134(3-4):277-286.

Re, M., and V. Barros. 2009. Extreme rainfalls in SE South America. Climatic Change 96(1):119-136.

Red de Monitoreo de las Aguas y del Territorio. 2015. http://RED-MATE.com.ar

Restovich, S. B., A. E. Andriulo, and S. I. Portela. 2012. Introduction of cover crops in a maize-soybean rotation of the Humid Pampas: Effect on nitrogen and water dynamics. Field Crops Research 128(0):62-70.

Salvagiotti, F., K. G. Cassman, J. E. Specht, D. T. Walters, A. Weiss, et al. 2008. Nitrogen uptake, fixation and response to fertilizer N in soybeans: A review. Field Crops Research 108(1):1-13.

SAS. 2008. SAS/STAT User's Guide. Version 9.2. SAS Institute Inc., Cary, NC. Pp. 846.

Sasal, M. C., M. G. Castiglioni, and M. G. Wilson. 2010. Effect of crop sequences on soil properties and runoff on natural-rainfall erosion plots under no tillage. Soil and Tillage Research 108:24-29.

Soil Survey Staff. 1999. Soil taxonomy, a basic system of soil classification for making and interpreting soil surveys. Agriculture Handbook Number 436. U.S. Department of Agriculture, Natural Resources Conservation Service. Washington D.C.

Thorup-Kristensen, K., J. Magid, and L. S. Jensen. 2003. Catch crops and green manures as biological tools in nitrogen management in temperate zones. Pp. 227-302. Advances in Agronomy. Academic Press.

Viglizzo, E. F., E. G. Jobbágy, L. Carreño, F. C. Frank, R. Aragón, et al. 2009. The dynamics of cultivation and floods in arable lands of Central Argentina. Hydrology and Earth System Sciences 13(4):491-502.

Zhang, Y. K., and K. E. Schilling. 2006. Effects of land cover on water table, soil moisture, evapotranspiration, and groundwater recharge: A Field observation and analysis. Journal of Hydrology 319(1-4):328-338. 